\title{
Una donna che parla alle donne: la Prima guerra mondiale vista da Matilde Serao in Parla una donna
}

\author{
Silvia ZANGRANDI \\ Libera Università di Lingue e Comunicazione - IULM (Milano) ${ }^{1}$ \\ silvia.zangrandi@ulm.it
}

Recibido: $18 / 05 / 2015$

Aceptado: 21/06/2015

\section{RIASSUNTO}

Tornare su uno scritto trascurato come Parla una donna (una raccolta di articoli usciti su Il Giorno da maggio 1915 a marzo 1916) di Matilde Serao significa considerare la guerra da un particolare punto di vista: quello femminile. Serao infatti non solo è donna lontana dai campi di battaglia e dalla vita in trincea, ma è donna che si rivolge alle donne. Se si accetta questa particolare prospettiva, il volume si rivelerà interessante sia per lo spaccato di vita sociale sia perché confermerà l'idea comune di come i giornalisti nascondessero $\mathrm{o}$ edulcorassero la realtà cruda della guerra. L'articolo analizza tre tematiche: la rappresentazione della guerra; la patria; lo status della donna. In essi si rintraccia la retorica sottesa che si traduce nella fierezza di essere italiani, nel dolore misto all'orgoglio delle donne che hanno al fronte un figlio, un marito, un fratello, nell'ardire dei soldati.

Parole chiave: Serao, Grande guerra, Patria, donne.

\section{A woman who talks to women: The First World War interpreted by Matilde Serao in Parla una donna (A Woman Talks)}

\begin{abstract}
A re-reading of Parla una donna (Woman's talk), the collection of Matilde Serao's articles that appeared in Il Giorno between May 1915 and March 1916, will help us re-consider the war from a woman's perspective. Indeed Serao is not only a woman far away from the battlefields and from the life of trench, but also a woman who speaks to women. If we accept this particular perspective, the book becomes interesting both as a portrait of social life and as evidence of the common belief that journalists hid or sweetened the harsh reality of war. The three main themes analysed in the articles - the representation of the war, the Homeland, the status of women - are characterized by an underlying rhetoric: the boast of
\end{abstract}

${ }^{1}$ Dipartimento di Letterature comparate e Scienze del linguaggio, Via Carlo Bo 1, I20143 - Milano. 
being Italian, the mixture of sorrow and pride of the women whose sons, husbands, brothers are at the front, the courage of soldiers.

Key words: Serao, Great War, Homeland, women.

SOMMARIO: 1. Introduzione 2. Matilde Serao e la guerra 3. Parla una donna 4. La rappresentazione della guerra nel volume di Matilde Serao 5. La patria 6. Lo status della donna 7. Conclusioni.

È inutile aspettare delle trasformazioni o dei rinnovamenti dalla guerra [...] come è inutile sperare che i letterati ritornino cambiati, migliorati, ispirati dalla guerra.

Renato Serra (1974: 526)

\section{INTRODUZIONE}

A cento anni dall'entrata dell'Italia nel primo conflitto mondiale, la rilettura di testi pressoché dimenticati può essere utile da un lato per esaminare con quale modalità scrittori e giornalisti raccontavano il conflitto ai loro contemporanei, dall'altro per dare, a distanza di anni, una nuova collocazione interpretativa agli scritti presi in esame. Questo studio intende occuparsi del volume Parla una donna a firma di Matilde Serao ${ }^{2}$. Il volume di Serao non ha ottenuto grande attenzione da parte della critica né dai suoi contemporanei né in tempi recenti ${ }^{3}$ : nelle monografie e negli studi a lei dedicati il commento al volume, nei casi migliori, si limita a poche laconiche righe; se si escludono il saggio breve di Elisabetta Rasy (Rasy 1994) e l'introduzione di Nunzia Soglia all'edizione anastatica del 2014, non vi sono studi specifici dedicati all'opera. Le ragioni sono molteplici: il lettore avverte un certo fastidio, un senso di insofferenza per la retorica di stampo patriottico di cui

${ }^{2}$ Il volume, pubblicato per la prima volta nel 1916 e ristampato nel 1921 sempre per i tipi di Treves (tutte le citazioni si riferiscono a questa edizione), raccoglie gli articoli che Matilde Serao pubblicò dal maggio 1915 al marzo 1916 sul quotidiano Il Giorno. Segnaliamo una copia anastatica del volume del 1921 per i tipi di Il Grappolo di Salerno a cura di Nunzia Soglia uscita nel 2014.

3 «Tra le ragioni del silenzio su questo libro mi sembra infatti che la principale possa essere la sua scarsa qualità e il fatto che o com'è probabile non era altro che una serie di articoli o rubriche giornalistiche rivisti e ricuciti insieme, o che comunque con la provvisorietà e la trasandatezza di un facile elzeviro è stato composto» (Rasy 1994: 244); «Parla una donna, per i suoi toni a volte troppo enfatici, forse non ha grande valore. Ne è consapevole anche l'autrice quando in prefazione avverte il lettore che non vi troverà "nessuna veste letteraria"» (Soglia 2014: 11). 
sono pervase le pagine di Serao; inoltre ci si avvicina al testo con un certo sospetto: il volume infatti si colloca tra quei lavori scritti da coloro i quali hanno vissuto da lontano la guerra, l'hanno, per così dire, combattuta da casa e quindi di essa non possono che avere «una visione distorta, idealizzata, del tutto prona alle aspettative del proprio orgoglio nazionalistico» (così si esprime Pannunzio commentando i racconti di Federico De Roberto che scrisse racconti riguardanti la Grande guerra pur non avendola combattuta: anche per lui «la trincea è solo un elemento lontano, [...] un non-luogo totalmente alieno»; Pannunzio 2000: 121). La particolare prospettiva che offre lo scritto di Serao è evidente fin dal titolo: la sua posizione eccentrica, intesa come fuori, lontana dal centro del conflitto in quanto donna, ci avverte da subito che non dobbiamo aspettarci le denunce che, al contrario, si leggono negli scritti di Slataper, di Serra, di Gadda, di Comisso, di Stuparich. «Alcuni libri di questa guerra, L'esame di coscienza di un letterato, Il giornale di guerra e di prigionia, Giorni di guerra, Guerra del '15, riusciranno davvero esemplari, tra i migliori di una nuova letteratura: una letteratura di esperienze significative, puntualmente vissute» (Bertacchini 1966: 476). Ne è un esempio il Giornale di guerra e di prigionia di Gadda edito da Einaudi nel 1965 che raccoglie anche un diario inedito, il Giornale di Campagna, nel quale Gadda, con violenta sincerità, inveisce contro i responsabili della «guerra-baraonda»: dai generaloni a Salandra e a «quello scemo balbuziente d'un re» (annotazione datata Edolo, 20 settembre 1915) a quei deputati che «vanno a veder le trincee» (annotazione datata Edolo, 20 settembre 1915) e infine ai furieri e ai marescialli che «ridono e sgavazzano» (annotazione datata Edolo, 6 ottobre 1915) mentre gli altri soffrono e «il loro martirio è senza nome» (annotazione datata Edolo, 6 ottobre 1915). È stato detto che la Grande guerra supera ogni possibilità di racconto (Gibelli 2003), tuttavia sappiamo che molti, anche chi aveva poca dimestichezza con la pagina scritta, sentì forte la necessità di tracciare nero su bianco le proprie esperienze e le proprie opinioni.

\section{MATILDE SERAO E LA GUERRA}

Tornare su uno scritto trascurato come Parla una donna assume un senso proprio se si vuole considerare la guerra da un particolare punto di vista: quello femminile. Serao infatti non solo è donna, come detto, lontana dai campi di battaglia e dalla vita in trincea ${ }^{4}$, ma è donna che, scrivendo di donne, si rivolge alle donne, è madre che si rivolge alle madri avendo anche lei al fronte tre figli (ai quali per altro dedica il volume). Se si accetta questa particolare prospettiva - che non

\footnotetext{
${ }^{4}$ Caso singolarissimo fu quello di Amalia Cottini Osta, conosciuta con lo pseudonimo di Flavia Steno, che, diversamente dalla Serao, abbracciò la causa dell'interventismo tanto da chiedere di essere mandata come corrispondente in Austria nel 1915. Steno fu fra le poche donne a vedere e a raccontare il conflitto al fronte e a scriverne sulle pagine di Il secolo XIX. Per approfondimenti si veda Freschi (2013).
} 
può che essere distorta proprio perché le vicende narrate non sono colte sul campo, non sono testimonianze dirette del conflitto ma da un lato sono considerazioni della Signora Serao ${ }^{5}$ sulla scorta delle notizie che arrivavano dal fronte e dall'altro sono suggerimenti riguardo alla condotta che le donne rimaste a casa avrebbero dovuto mantenere - allora il volume si rivelerà interessante sia per lo spaccato di vita sociale che contiene sia perché la lettura confermerà l'idea comune di come $\mathrm{i}$ giornalisti nascondessero o edulcorassero la realtà cruda della guerra. Prezzolini definì «sistema di bugie» quello che leggeva sui giornali: «i corrispondenti di giornali in generale sono stati particolarmente bugiardi. I soldati li hanno presto odiati» (Prezzolini 1919: 45, 47). A proposito dei comunicati che venivano redatti quotidianamente, che utilizzavano aggettivi eufemistici, come «accanito» e «vivace» (ad esempio: «il nemico fu respinto dopo vivaci combattimenti», oppure «vi fu una accanita rappresaglia»), Paul Fussell ci dice che il matematico Alexander Aitken, che combatté la prima guerra mondiale e fu ferito sulla Somme, «imparò che accanito e vivace avevano significati ben precisi: significavano che circa metà della compagnia era stata uccisa o ferita durante un'incursione» (Fussell 2000: 223). Secondo Fulvio Senardi:

c'era chi voleva continuare a sognarla, e a farla sognare, sull'arcaico copione di duelli a cavallo con le sciabole scintillanti al sole, o immaginava le morti in prima linea con i caduti compostamente adagiati sul terreno [...]. Illusioni alimentate [...] dai media dei paesi coinvolti nel conflitto, che mettono a punto sofisticati strumenti di produzione del consenso volti a influenzare [...] la pubblica opinione. (Senardi 2008: 28)

Sull'argomento lungamente si intrattiene Paul Fussell: secondo lo studioso le persone rimaste a casa difficilmente avrebbero potuto avere la giusta percezione di quanto accedeva al fronte per ragioni attribuibili non solo alla reticenza della stampa, colpita dalla censura, ma anche dalle condizioni di quella guerra che erano nuove rispetto ai conflitti che l'avevano preceduta. Inoltre i soldati che scrivevano dal fronte evitavano di dire davvero la verità per non allarmare più di quanto già lo fossero le famiglie a casa. E ancora Fussell nel suo studio cita la lettera di un soldato scritta nel 1917 alla sua famiglia in cui si legge: «penso spesso quanto sia strano che la vita domestica proceda tranquilla a Weybridge, e in qualsiasi altra parte dell'Inghilterra, mentre per tutto il tempo qui accadono cose spaventose» (Fussell 2000: 80). Ancor più forte è quanto scrive Carlo Salsa: «non so perché mi sia stupito di trovare tanta gente indifferente per le vie [...]. Qui non c'è la guerra: la guerra è lassù, per noi, nell'altro mondo: qui cosa ne sanno» (Salsa 1982: 200). Salsa, arruolato in fanteria, inviato al fronte all'inizio della guerra e fatto prigioniero nel 1917, nel suo libro riferisce dell'insofferenza da parte dei soldati che tornano dal fronte, perché malati o perché è stato loro concesso un periodo di

\footnotetext{
${ }^{5}$ Serao era semplicemente chiamata la Signora dalle persone che lavoravano presso $I l$ Giorno.
} 
congedo, nei confronti dei civili restati a casa perché questi ultimi sono incapaci di capire cosa hanno provato.

\section{PARLA UNA DONNA}

In questo clima si inserisce il volume di Matilde Serao Parla una donna: come detto, si tratta di articoli usciti su Il Giorno, il quotidiano da lei fondato nel 1904, apparsi a partire dal 25 maggio 1915, primo giorno di entrata in guerra dell'Italia, a marzo 1916 (la data non viene precisata), raccolti in volume nel 1916. È giusto sottolineare che il giornalismo di fine Ottocento-inizio Novecento rispondeva alla richiesta di conoscenze di una società di recente formazione borghese per la quale $\mathrm{i}$ quotidiani potevano offrire spiragli sul mondo. L'articolo di presentazione del nuovo giornale è da considerarsi programmatico:

il fremito affettuoso della folla non ci domandava solo [...] una lunga e minuziosa cognizione dei fatti accaduti in tutto il mondo, cioè uno di quei notiziari universali aridi di ogni pensiero $[\ldots]$ ma ci chiedeva qualcosa di $[\ldots]$ necessario alla vita sociale $[\ldots]$ la difesa nobile e pura della coscienza [...] la edificazione di un mondo morale più alto. (Trotta 2008: 161)

In esso Serao darà vigore alla sua vocazione sociale e pedagogica ed è in questa accezione che vogliamo leggere Parla una donna. La sua narrativa, per dirla con Banti, è «un sentimento mosso dall'osservazione» (Banti 1965: IX): il volume che raccoglie i suoi articoli è un libro-documento che offre uno spaccato sociale dell'epoca, autentica letteratura di denuncia, ma in esso Serao trascura la verità documentaria. Non si può infatti nascondere che manca la registrazione lineare dei fatti: le battaglie sull'Isonzo sono totalmente taciute (si trovano infatti solo rari accenni: «l'Isonzo, arrossato da tanto sangue!»; Serao 1916: 167), «il Carso [...] resterà memorabile nella storia della guerra europea, come il più alto documento del valore italiano» (Serao 1916: 296), della battaglia di Verdun del 21 febbraio 1916, una delle più lunghe e più sanguinose della prima guerra mondiale, viene fatto un cenno rapidissimo («il cannone furioso di Verdun»; Serao 1916: 304). Si rilevano genericità e vaghezza riguardo ai luoghi geografici dove si svolse il conflitto: «dagli ultimi giorni di maggio, per cinque mesi [...] per conquistare questa terra, questi monti e questo mare, sono caduti i nostri prodi» (Serao 1916: 217), le Alpi Giulie e quelle Carniche sono solo nominate (Serao 1916: 295) e non vengono aggiunti particolari; la questione delle terre irredente si risolve in una vaga allusione: «i nostri soldati si battono da eroi, e ci conquistano le terre che dovevan essere nostre» (Serao 1916: 267). Ciò provoca in noi sospetto: pur nell'aspirazione all'autenticità Serao si presenta come interprete fedele della realtà proprio nella sua funzione di giornalista e in una lettera che scrisse all'amico Gaetano Bonavenia scrive:

io intendo per realismo, la vita tutta, tutta con la sua poesia altissima, con la sua modesta prosa, coi suoi slanci generosi e con le sue meschinità reali [...] Nasce invece il realismo 
nel suo vero, nel suo ampio senso, lo studio dell'uomo come corpo e come spirito. Nel mio articolo del Novelliere intitolato "Vita nuova" ci è la professione di fede. (Serao 1938: 404)

ella resta in superficie, non si occupa del dato cronachistico (è noto che i suoi figli che collaboravano al giornale del padre, Edoardo Scarfoglio, aggiungessero allo slogan «Chi legge Il Giorno sa tutto», "Chi legge Il Giorno sa tutto... ventiquattr'ore dopo!») e nel suo approccio alla realtà della guerra finisce per ridurre la complessità del reale a cenni vaghi e indeterminati. Scrive nella Prefazione che la folla chiede allo scrittore di «esaltare questo intelletto col dolore, vergando delle parole immortali sulla guerra», ma lo scrittore è impotente di fronte a questa immane tragedia: «dove, dove sono le vaste parole colorite, smaglianti, per descrivere quest'oceano di sangue senza sponde?». Allo scrittore, conclude Serao, non resta che dichiarare amaramente: «esiste la guerra: ma è una realtà senza parole: ma è una tragedia senza poeta» (Serao 1916: XI). Durante la lettura capiamo che, in sintonia con quanto chiedevano i governi - non solo quello italiano -, l'intento è quello di non offrire immagini allarmanti e di sviare l'attenzione su argomenti paralleli ma meno angosciosi. Tra i diversi argomenti toccati da Serao (la pace; il Re e la famiglia reale; l'importanza della preghiera ...) qui mi occuperò di tre tematiche: la rappresentazione della guerra; la patria; lo status della donna. In essi si rintraccia la retorica sottesa a questa guerra che si traduce nella fierezza di essere italiani, nel dolore misto all'orgoglio delle donne restate a casa, nel coraggio e nell'ardire dei soldati, in perfetta sintonia con la canzone pressoché dimenticata Italia bella: «E sulla terra e sul mare / S'udì gridare: / Evviva il Re! / Italia bella fiorente e forte / Sorriso eterno di primavera» ${ }^{6}$.

\section{LA RAPPRESENTAZIONE DELLA GUERRA NEL VOLUME DI MATILDE SERAO}

È persino pleonastico dire che «guerra» è il termine che ricorre con più frequenza in questo volume. È noto che Matilde Serao fosse contraria all'intervento in guerra da parte dell'Italia e in diverse occasioni aveva apertamente dichiarato la propria neutralità ${ }^{7}$. Con la prolissità verbosa tipica della sua prosa, sin dall'apertura

${ }^{6}$ Di questo canto non è certa la data di composizione, in ogni modo si pensa al 1918 perché nella prima strofa fa riferimento alla bandiera tricolore che sventola nel cielo di Trieste, città che fu annessa all'Italia proprio in quell'anno.

${ }^{7}$ Benché in Parla una donna Serao sia chiaramente antibellicista, non così si può dire a proposito della guerra in Libia del 1912 in cui esaltò l'impresa: nell'articolo Evviva la guerra scrive che ora gli italiani «si sentono uniti in una sola idea, uniti in un solo desiderio, la fortuna, l'onore, la vittoria, la gloria dell'Italia». In realtà, come sostiene Tommaso Scappaticci, «l'esaltazione dell'impresa di Libia [...] non indulge tanto al motivo 
del volume sciorina verbi e sostantivi per descrivere la guerra, che «arde, divampa, distrugge» ogni cosa (Serao 1916: IX): i «campi [sono] flagellati dalla mitraglia»e il «fremito lungo di angoscia» (Serao 1916: X) affligge chi è restato a casa. Il suo sentimento non cambierà col tempo, tanto è vero che in uno degli ultimi articoli datato febbraio 1916, rivolgendosi ai bambini, scrive: «o piccole anime, possiate, voi, quando ingrandirete, nulla ricordare, nulla, di tutte queste cose orrende!» (Serao 1916: 309). Da giornalista, però, si trova obbligata a parlare del conflitto e, in sintonia con quanto imponeva la censura, il cui intento era ovviamente di evitare che la realtà della guerra fosse conosciuta nella sua interezza e brutalità, rimarca, tramite aggettivazione enfatica e obiettivamente irritante perché poco credibile, il valore e l'eroismo dei soldati. Ricordando nell'articolo dell'autunno 1915 (manca la data precisa) il conferimento a cinque soldati di una medaglia d'argento al valor militare da parte del re, così scrive:

chi eran mai costoro che il Re indica alla Nazione e sul cui petto si posa un fregio di argento, che sarà il più prezioso fra $\mathrm{i}$ tesori della loro vita? [...] venivano da quella immensa massa di uomini [...] che ha formato una muraglia di acciaio con i suoi petti e la sua volontà $[\ldots]$ questa possente forza sconosciuta. (Serao 1916: 159)

E per descrivere la loro «epica ventura» dice che

ognuno di costoro si espose volontariamente e animosamente a un pericolo estremo [...] seguitò ad avanzarsi sotto il fuoco nemico fulminante, pur di condurre a termine una sua audace impresa $[\ldots]$ feriti, sfiniti, non si mossero, sinché non venne l'ordine del loro ufficiale. (Serao 1916: 160)

Conclude il lungo articolo sottolineando ulteriormente «quanto eroismo ignoto, oscuro, tacito, ha sollevato queste anime semplici di popolani, di operai, di contadini, e le ha elevate in una vita superiore» (Serao 1916: 162), facendo «trabalzare di ammirazione» (Serao 1916: 163) la stessa Serao.

Come detto in precedenza, stupisce la mancanza di menzione delle battaglie che tra il ' 15 e il ' 16 i soldati italiani combatterono, mentre insistentemente viene evidenziata l'audacia e il coraggio dei soldati, della

mirabile, impetuosa gaiezza, con cui il soldato napoletano si è gittato sul nemico, e quante e quante volte il suo ardore ha dovuto esser frenato dai suoi ufficiali, che gli gridavano di star quieto, di star tranquillo, di aspettare gli ordini [...] giacché questo è il nostro soldato [...]: un superbo e cosciente istrumento di guerra. (Serao 1916: 39)

Poche pagine dopo si lancia in un autentico inno al soldato napoletano tra le pp. 39 e 43, che tocca il suo apice a p. 41: «buon soldato napoletano, che sai batterti

nazionalistico $[\ldots]$ ma si risolve nella prospettiva della spedizione utile a servire da antidoto alla lotta di classe e a realizzare la concordia sociale» (Scappaticci 1995: 275). 
[...] sino al sacrificio [...] tu che sai toccare le cime dell'eroismo [...] tu che sai combattere, e vincere e morire» (Serao 1916: 41). Frequentemente Serao menziona le classi sociali più umili, e in particolare i contadini che hanno dovuto lasciare la campagna per andare a combattere, e di loro tesse sempre elogi: «i contadini d'Italia sono partiti per la guerra [...] e si sono battuti [...] con un impeto e con una tenacia così mirabile, che i loro comandanti ne fanno, ancora e sempre, gran lode» (Serao 1916: 15). Non dimentica, ovviamente, di parlare di singoli ufficiali, senza nominarli proprio perché in questo modo il singolo assume valore per tutti: «egli narra le pruove di supremo valore della sua compagnia, che aveva attaccato il nemico, alla baionetta, e che aveva fatto prigionieri novantacinque soldati austriaci e un ufficiale» (Serao 1916: 65); in un altro articolo parla poi di un ufficiale di marina che «chiedeva di correre il maggior rischio, pur di poter attaccare e affondare il nemico» (Serao 1916: 86); i soldati sono spesso definiti «formidabili» (Serao 1916: 296), «incomparabili» (Serao 1916: 295); inneggia alla flotta italiana: «diventa, o flotta italiana, formidabile, anche per avere atteso, anche per aver avuto pazienza [...] e ogni tua nave valga tre del tuo nemico e ogni tuo uomo valga cinque dei tuoi nemici» (Serao 1916: 91-92).

Serao fa riferimento alle cartoline che gli uomini dal fronte inviavano alle loro famiglie. Robert Musil scrive nei suoi Diari che gli italiani in special modo provano una particolare "voglia bellica": "cartoline illustrate [...] ritraggono i loro soldati nelle pose eroiche preferite; sono ancora rimasti al gioco dei soldati» (Bertacchini 1966: 476). Serao conferma l'opinione di Musil riassumendo il contenuto di una di queste cartoline in cui un giovane prega «la madre di non stare in pensiero per lui, spiegando alla madre che la guerra si sta vincendo [...] narrando [...] che gli austriaci scappano sempre» (Serao 1916: 43) (siamo nel luglio del 1915). Questo accenno al mezzo di comunicazione usato nel primo conflitto mondiale è interessante per due motivi: il primo è che ci mostra come veniva considerato il nemico, che è sempre descritto in posizione di inferiorità rispetto al soldato italiano: è pavido, è in fuga, è disorganizzato. Paul Fussell, a proposito della percezione del nemico, scrive:

possiamo definire dicotomizzante un permanente abito mentale dell'età moderna, che sembrerebbe possibile far risalire alla realtà della Grande Guerra. "Noi" siamo individui con nome e identità personale; esso è soltanto un'identità collettiva. Noi siamo visibili; esso è invisibile. Noi siamo normali; esso è grottesco. Le cose che ci appartengono sono naturali; le sue, strane. Il nemico non è buono come lo siamo noi. (Fussell 2000: 97)

Il secondo motivo è che, così scrivendo, Serao mostra in quale misura le persone rimaste a casa venivano informate: sia $\mathrm{i}$ giornali sia gli uomini in guerra erano reticenti nel raccontare i fatti nella loro completezza perché non volevano allarmare le famiglie; a questo si aggiungeva la censura, che imponeva loro di dire solo alcune cose: il risultato era una notizia frammentata, imparziale e scorretta ma che solo alla fine del conflitto si sarebbe scoperta nella sua falsità. 
Si diceva che gli articoli di Matilde Serao sono interessanti perché ci offrono uno spaccato di vita sociale dell'epoca, e forse proprio questa particolare prospettiva rappresenta per noi l'aspetto più interessante dell'intero volume. Pur non dimenticando il conflitto in atto, Serao ci narra di Piccole storie di guerra (Serao 1916: 282-292), con l'intento di dare notizie frivole (noi oggi parleremmo di gossip) per distogliere il lettore dall'angoscia quotidiana. Racconta quindi la storia di un giovane ufficiale, diventato muto a causa di una bomba scoppiata nelle vicinanze, che torna a casa in licenza, nella convinzione che, ritrovando la fanciulla che aveva conosciuto un mese prima di partire, avrebbe riacquistato il dono della parola. Purtroppo, una volta tornato a casa, scopre che la ragazza tante volte sognata mentre era in trincea si era unita a un altro uomo e tale scoperta lo sconvolge più ancora della bomba che era caduta accanto a lui. La giornalista racconta poi la storia del sottotenente ferito gravemente che si innamora di un'infermiera e pensa di essere corrisposto fin quando non scopre che lei è già sposata e, a causa delle ferite in battaglia e del dolore causato dalla scoperta, muore; oppure la storia della ragazza che non sopporta il pensiero di sapere il suo uomo in battaglia perennemente in pericolo e muore per l'affanno proprio mentre lui ritorna a casa sano e salvo. La guerra è ritratta anche come occasione di incontro: nell'articolo Matrimoni di guerra si parla del boom dei matrimoni tra aprile e maggio 1915, nell'imminenza dell'entrata in guerra, quasi si volessero esorcizzare i pericoli e le paure legate al conflitto con la realizzazione di un progetto di vita comune. Serao pensa che la giovane moglie non potrà «odiare la guerra. Giacché è per essa che ella ha incontrato l'uomo che le deve essere compagno; è per essa che ella sarà felice: felice anche nell'attesa» (Serao 1916: 174). Naturalmente, però, non può tacere le tante, troppe vittime della guerra. Frequentemente parla del dolore delle donne che hanno perso un figlio, un marito, un parente in guerra e di quelle che, strazio ancora più grande, non hanno potuto seppellirlo: «più torturante, più spasimante sarà il dolore delle madri, che dettero un figlio alla patria e che non potettero riavere né riabbracciare il cadavere [...] tremenda e ineluttabile legge della guerra!» (Serao 1916: 219). L'immedesimazione con la disperazione di queste donne fa esclamare alla giornalista: i «caduti in guerra, i morti senza tomba [... sono i] nostri salvatori» (Serao 1916: 221). Non si può tacere che certa retorica infastidisca il lettore contemporaneo; prendiamo a esempio questa affermazione difficilmente credibile: «nella nostra nobilissima guerra $[\ldots]$ sono caduti i nostri prodi soldati $[\ldots]$ e sono giaciuti, morti, $[\ldots]$ coi loro corpi composti nella più bella fra le morti» (Serao 1916: 217).

Infine, spesso si occupa dei soldati feriti: ci parla della «stazione di Roma, ove, ogni giorno, ogni ora, da mesi e mesi, è un continuo, vertiginoso, vorticoso movimenti di soldati e di ufficiali, di feriti che giungono e di guariti che ripartono [...] un vero quadro impressionante di umanità in guerra» (Serao 1916: 307). Si dilunga nel dare particolari sui tanti feriti che vede o di cui sente parlare e sempre questo rappresenta l'occasione mettere in luce l'eroismo dei nostri soldati: 
malgrado la mano destra fasciata, malgrado la testa fasciata [...] malgrado la gamba che si trascina, brillanti di gioventù, tutti, malgrado che sieno feriti e che vengano a curarsi, questi ufficiali [...] raccontano i più commoventi episodi di guerra, gli eroici episodi. (Serao 1916: 62-63)

\section{LA PATRIA}

Uno spazio di tutto rilievo in Parla una donna è occupato dalla nozione di patria, anzi è bene parlare di autentico culto della patria che può essere riassunto in queste poche righe: «la bella, sana e vigorosa Italia, che non ha tara, che non ha pecca, che è intiera, florida, splendida di salute morale e fisica!» (Serao 1916: 15). L'amor patrio è «ardentemente sentito» sin dalle prime battute (Serao 1916: XIII) e spesso in Serao si confonde «l'amor del mio paese [con] l'amor dei miei figli» (Serao 1916: XIV). Durante la lettura si evince il senso di appartenenza alla patria costituita attraverso valori che definiscono l'essenza stessa della comunità: «questa incomparabile Italia» (Serao 1916: 201), «questa terra porta l'augusto nome di patria, ed è quanto di più alto componga, attorno a noi e in noi, il senso della umana dignità; poiché essa ci rese fieri di essere uomini e cittadini» (Serao 1916: 7). Ecco quindi che «l'uomo a cui la patria domanda il sangue e la vita, contrae un legame che è immagine di lunghi anni pacifici e laboriosi [...] e forse [...] quest'uomo avrà dato la sua vita alla gloria del suo paese» (Serao 1916: 170). Con stucchevole retorica, il senso di patria e il «civile coraggio» (Serao 1916: 161) dei soldati vengono messi in risalto dalla dedizione con la quale essi combattono: «ognuno di costoro si espose volontariamente e animosamente a un pericolo estremo [...] seguitò ad avanzarsi sotto il fuoco nemico fulminante, pur di condurre a termine una sua audace impresa» (Serao 1916: 160). L'esaltazione dell'eroismo sacrificale si evince quando, parlando delle donne che vedono i loro figli partire, esclama: «per loro era cosa naturalissima che i figli, unico sostegno di quelle grame esistenze, servissero la patria» (Serao 1916: 95), e ancora: «avevate soltanto quell'unico figliuolo e generosamente, l'avete dato alla patria» (Serao 1916: 168). Consapevole delle enormi perdite di vite umane, si rivolge sia alle madri sia alle vedove nel tentativo di lenire la loro disperazione, mostrando la morte da eroe del soldato: «il figliuolo è morto in un atto di eroica dedizione alla patria» (Serao 1916: 144); «ella sarà la più fedele fra le vedove, fedele a colui che seppe onorare la sua patria e la donna che amava» (Serao 1916: 173). Il senso di appartenenza a un'unità comune viene sottolineato anche dal fatto che la giornalista ci dice che «con curiosità e con orgoglio i nostri occhi seguono [...] i premi assegnati a coloro che hanno dato tutto il loro entusiasmo e, spesso, il loro sangue, e, spesso, la loro giovanissima vita per la patria» (Serao 1916: 249).

Famiglia e patria si confondono e si fondono nel pensiero di Matilde Serao, ma patria per lei rima anche con Re: «quello che più ci fa obbliare il sangue italiano che scorre, e ogni rude sacrificio [...] è questo connubio così perfetto fra il Re e l'Italia, e il popolo italiano» (Serao 1916: 238). Per Serao, fervente monarchica, amica della 
nobiltà, «Vittorio Emanuele di Savoia, [è] principe ereditario di un regno nuovo ma grande, ma possente» (Serao 1916: 306). (Serao amava trascorrere brevi periodi in montagna per poter osservare da vicino l'aristocrazia legata alla monarchia ma, d'accordo con De Nunzio Schilardi (1986), la fede monarchica più che una scelta ideologico-politica, è un'adesione istintiva e piccolo-borghese alla famiglia reale).

Infine, la nozione di patria coincide con la tanto sospirata pace, una pace che però non deve scendere a compromessi : «noi vogliamo la pace a fronte alta, la pace della vittoria, del buon dritto, della dignità trionfante!» (Serao 1916: 313). La pace deve essere «onorata e vantaggiosa per il nostro paese, è la meta a raggiungere la quale, tendono i nostri sforzi e i sacrifici nobilissimi dell'esercito e dell'armata». Attorno al termine «onorata» si rivolge l'attenzione di Serao e questo termine viene più volte ripetuto: «questo sacrificio non è vano, poiché esso ci condurrà a una pace onorata e vantaggiosa, in cui l'Italia vedrà, anzi tutto, cresciuta infinitamente la sua dignità di nazione e aumentato il suo territorio, di terre che fatidicamente eran sue» (Serao 1916: 241-242). Insomma, Serao identifica con il termine patria il senso di lealtà e di affetto del popolo nei confronti del proprio paese, associando l'idea di nazione con quella di libertà e onore. Si spiega così il fatto che Serao non si espone nel chiedere la pace e, riportando il grido sgomento del Papa che invoca la pace e la fine del conflitto, lascia a lui solo il compito di chiederla con forza, motivando così le ragioni:. «se noi, uomini e donne, la chiediamo pubblicamente, ci chiameranno vili e traditori [...] Ma voi, Santo Padre, siete fuori e sovra ogni interesse [...] potete pregare, chiedere, proporre, senza umiliarvi» (Serao 1916: 125-126).

\section{LO STATUS DELLA DONNA}

D'accordo con Anna Banti, si legge la produzione di Matilde Serao «spesso con irritazione, talvolta con ammirazione» (Banti 1965: IX) e questo avviene anche durante la lettura di Parla una donna. Del volume ammiriamo la forza con la quale proclama la centralità della donna che combatte da casa una battaglia quotidiana contro le difficoltà sorte dalla mancanza degli uomini impegnati al fronte: in «queste pagine $[\ldots]$ son notati gli episodi della virtù muliebre, $[\ldots]$ sono espressi i sensi di ammirazione, per tanto valore di fanciulle, di donne, di madri» (Serao 1916: XV). Serao è in sintonia col suo pubblico, soprattutto quello femminile e meridionale del quale conosce vizi e virtù. Si infervora quando scrive che in Italia le donne che si adoperano in tanti settori come l'arte, la scienza, la carità

non possono aver ricompensa, di nessun genere, giacché queste ricompense non esistono e non sono mai esistite. Non un ordine cavalleresco, per le donne, non un ordine religioso, non una medaglia, non un premio, nulla di nulla, mai, niente di niente. Lo Stato italiano ha mostrato [...] la totale indifferenza, per la mente e per l'anima feminile. (Serao 1916: 251) ${ }^{8}$

\footnotetext{
${ }^{8}$ Si riporta fedelmente la scelta ortografica di Serao che rispecchia la grafia del tempo.
} 
Contemporaneamente siamo infastiditi dal fatto che alle donne affidi solo la funzione familiare e mai quella pubblica: per lei le donne non devono entrare in politica perché non hanno opinioni ma sentimenti! Per dirla con Wanda De Nunzio Schilardi:

la Serao, in linea con l'ideologia cattolico-moderata elaborata nella prima metà dell'Ottocento, riconosce un ruolo tutto domestico alla donna predicando la santità del matrimonio e celebrando i motivi della solidarietà e della collaborazione. (De Nunzio Schilardi 2005: 120)

Scrive Serao su La lega della Democrazia il 21 maggio 1880:

per la vita, per l'amore, l'arte ci vuole la donna. Istruita, ma donna. Maestra del popolo, infermiera, scrittrice, educatrice, ma donna. Niente diritti politici, niente ingerenze elettorali, niente attribuzioni maschili, niente professioni impossibili [...] per semplice curiosità, senza nessun interesse, senza nessuna ammirazione, leggo delle socialiste $[\ldots]$ sono casi patologici, deviazioni del cervello che soffoca il cuore, aberrazioni del senso femminile. (Masini 1965: 253)

In Parla una donna si schiera contro il movimento delle suffragette in Inghilterra, che definisce "povera milizia feminista» che si adopera "perché le donne avessero il voto, vote for women, perché tutte le professioni e tutti i mestieri maschili si schiudessero alle donne» (Serao 1916: 232-233). A dimostrazione della sua sfiducia verso le richieste delle suffragette, scrive che:

in molte professioni, in molti uffici, le donne introdottevisi, hanno fatto un fiasco solenne $[\ldots]$ le tranviere di Roma sono odiate dalla popolazione dell'Urbe, perché sono inette, e molto più sgarbate dei tramvieri maschi. (Serao 1916: 235)

La sua dichiarazione esplicitamente contraria al movimento di emancipazione femminile palesa la sua visione conservativa del ruolo della donna: Serao non perde occasione per sottolineare la necessaria condizione di sottomissione e di debolezza della donna, ma questo suona contraddittorio in una donna come lei che era riuscita ad affermarsi in ruoli che a quei tempi spettavano esclusivamente agli uomini! Questa mancanza di coerenza disturba il lettore di oggi che, tuttavia, non può non riconoscerle la volontà di testimonianza della condizione femminile e, posizione modernissima, il coraggio di sostenere la necessità di una rivoluzione maschile attraverso la quale giungere a una migliore dignità e qualità della vita della donna. Insomma, per Serao la donna deve mantenersi all'interno della più rigida «tradizione femminile, ma curiosamente intesa come valore in sé, e non in rapporto all'uomo» (Rasy 1994: 250).

È opinione comune che la donna sia stata un soggetto estraneo alla Grande guerra, adatta solo ad assistere i feriti: le donne potevano confrontarsi con essa limitandosi a manifestare la loro pietas. Per dirla con Gibelli, la Grande guerra fu 
guerra femminile perché pretese il contributo materiale delle donne unito ai valori e ai simboli che il mondo femminile recava con sé (Gibelli 2002: 557). Come vedremo anche in Parla una donna, molte donne erano impegnate nei comitati di assistenza e di propaganda in cui fornivano un servizio sociale alla patria (nasce proprio durante la prima guerra mondiale a Milano la prima scuola per assistenti sociali). Inoltre, in ogni Comitato municipale di assistenza e propaganda esisteva una commissione di corrispondenza che aveva il compito di mantenere i contatti tra i combattenti e le loro famiglie (Molinari 2014: 468). Alcune donne, poi, svolgevano attività di madrine di guerra con la quale, tramite la corrispondenza con i soldati al fronte, davano loro un sostegno morale. Insomma, ovunque veniva trasmesso un modello di «donna tradizionale, rispettosa delle regole imposte dalla classe borghese e ben pensante, e un ruolo esclusivamente domestico e familiare» (De Nunzio Schilardi 2004: 25). Persino Flavia Steno, pur documentando dal fronte gli orrori della guerra, trasmise, come Serao, lo stesso modello di donna e infatti, nel 1915, scrisse su Il secolo XIX:

accanto al letto di un ferito ogni donna si sente madre: non sorella, non sposa, non compagna: madre! [...] il ferito, il malato le appartengono di diritto: ella ne rivendica la protezione quando non può rivendicarne l'assistenza materiale. (Freschi 2013: 65)

E a sua volta Serao, nell'articolo intitolato $L$ 'arte di sorridere, scrive:

quante donne io ho visto, negli ospedali, nelle ambulanze, curvarsi, con un sorriso mite, sui letti ove pativa un ferito [...] quest'arte del sorriso muliebre è adesso [...] un puro esercizio di coraggio e di pietà. (Serao, 1916: 196-197)

Secondo Augusta Molinari:

la storiografia italiana ha dedicato scarso interesse alla mobilitazione femminile [dal momento che] la mobilitazione civile per la guerra è stata studiata più nei suoi aspetti ideologici e politici che in quelli sociali [...] l'unico testo utile per avere un quadro della mobilitazione femminile nella Grande guerra, era quello pubblicato nel 1917 da Paola Baronchelli Grosson. (Molinari 2014: 467)

Questa giornalista, nota col nome di Donna Paola, operò nello stesso periodo in cui scrive Matilde Serao e intervenne sull'argomento, sottolineando l'indole pietosa e soccorrevole delle donne, sostenendo che la donna doveva «serbare della femminilità tutto quanto è soavità, dolcezza, pietà [...] insomma, quella "maternità" che è in tutte le donne» (Donna Paola 1915: 3-13) ${ }^{9}$. Nella sua indagine Molinari

${ }^{9}$ Donna Paola, al secolo Paola Baronchelli Grosson, fu giornalista (esordì nel 1895) e caporedattrice e collaborò a diversi quotidiani e riviste. Allo scoppio della prima guerra mondiale fu fervente interventista e a favore della partecipazione diretta delle donne nel 
dimentica di annoverare il volume Parla una donna di Serao che in realtà entra a pieno titolo tra gli scritti in cui la rappresentazione delle attività delle donne durante la prima guerra mondiale e il loro conseguente ruolo sociale sono ben delineati, vero punto di interesse del volume oggetto di questa indagine insieme a un più generale spaccato di vita sociale. Serao si rivolge alle donne di tutti i ceti sociali, chiede il loro impegno sociale e le associa nel dolore e nell'angoscia:

nobili dame, le cui mani tremanti schiudono il libro delle preci [...] borghesi [...] curve sul loro rosario $[\ldots]$ popolane, che sono inginocchiate sul nudo e freddo marmo che non hanno né libro né rosario [...] è a costoro, mie sorelle, carissime ignote, ma mie sorelle di pena, $[\ldots]$ che voglio dire, qui, la più schietta e più fraterna parola. (Serao 1916: 2)

Non nasconde le difficoltà che il conflitto porta con sé:

il cibo diventa caro, il carbone diventa caro, il lavoro scarseggia: sono le inevitabili conseguenze della guerra. Ma tu sei qui: [...] tu diminuirai tutto il tuo lusso, se sei una gran signora, perché esso vada a soccorso dei miseri; tu farai delle serie economie, in casa, se sei una borghese, per poter aiutare, anche più, coloro che patiscono; tu soccorrerai la più povera di te se sei una popolana. (Serao 1916: 287-288)

Pur nel distinguere ruoli e abitudini, accomuna tutte le donne in un'unica condotta: sia che si tratti di nobildonne sia che si tratti di popolane, Serao chiede loro moralità e contegno esemplari:

da che la guerra nostra è scoppiata, il mio cuore tremante di emozione ha raccolto episodi di tale gentilezza, di tale pietà, fra le famiglie della piccola borghesia, fra donne del popolo, che io vorrei poterli narrare e sono troppi. (Serao 1916: 33)

Per evidenziare il diverso comportamento delle donne italiane rispetto alle donne d'oltralpe, ci narra di quanto accade nei paesi coinvolti nella guerra come la Francia, la Germania, l'Inghilterra. Nell'articolo intitolato Amoreggiamenti racconta delle donne che si innamorano dei prigionieri tedeschi e, dopo essersi domandata se tale condotta sia opportuna, ricorda la storia di Giulietta e Romeo e conclude che «spesso è questo amore che ha condotto a una riconciliazione durevole» (Serao 1916: 71). Questa è l'occasione per elogiare le donne italiane che «si sono astenute dal filare [...] la donna italiana vale meglio, molto meglio, delle donne delle altre nazioni. Pure, la pietà che hanno avuta dei prigionieri austriaci le nostre donne, è stata molta» (Serao 1916: 73). Fenomeno prettamente francese, e solo in maniera minore tedesco, italiano e inglese, è quello delle indovine: si tratta di donne disoccupate che «si offrono alla curiosità e alla intima ansietà di chi ha persone in guerra» (Serao 1916: 180). Benché queste donne sfruttino la

conflitto nella speranza che ciò avrebbe potuto permettere alle donne il giusto riconoscimento politico e sociale. 
disperazione di chi è rimasto a casa e vorrebbe avere notizie del proprio caro in guerra, Serao non le giudica male (non dimentichiamo che è napoletana e la smorfia, la potenza degli amuleti, la superstizione sono parte integrante della cultura della città): «anche la illusione è immensamente necessaria, adesso» (Serao 1916: 181).

Ci racconta anche il fenomeno delle vedove bianche, ovvero di quelle donne che, pur non essendo state sposate ma solo fidanzate e il loro uomo è morto in combattimento, si sentono vedove. Serao diffida di queste donne che «obbedirono alla esaltazione, che viene da una terribile notizia di una morte in guerra» (Serao 1916: 141) e dice che il loro fidanzato «finirà per diventare un loro nemico intimo [...] a un tratto, bruscamente, gitteranno via tutto il loro passato, che, in verità, era fittizio, era tutta una falsità sentimentale» (Serao 1916: 142). E ancora, mentre ci parla di un fenomeno diffuso durante la guerra, ovvero il marrainage in Francia ${ }^{10}$, non nasconde la sua poca fiducia in questo servizio, aggiungendo che «in Italia [...] questo madrinaggio non è apparso né utile né necessario» (Serao 1916: 47) perché in Italia c'è il culto della famiglia e non c'è soldato che si senta solo e che resti abbandonato. Certo, spetta alle donne il compito di essere consolatrici: «Donne d'Italia, ci fu data una croce, la guerra: solleviamola, con coraggio, con forza, ed essa ci sembrerà un austero dono, se possa rendere più gloriosa la patria italiana» (Serao 1916: 6).

Un altro fenomeno di cui ci parla Serao è quello dell'opera collettiva femminile della quale nutre «forte scetticismo sull'efficacia» (Serao 1916: 18): quando quindici, trenta e più donne si aggregano, secondo Serao difficilmente trovano un accordo e così sprecano tempo. Dall'associazionismo femminile, dai protettorati femminili «non ne verrà fuori nulla di buono e di utile» (Serao 1916: 21), meglio l'opera personale, individuale. Torna sull'argomento nell'autunno del 1915, a proposito delle dame di provincia, «dame disoccupate, il cui unico bisogno [...] è stato quello di formarsi in tre, in quattro, in otto comitati [...] per gli scopi più bizzarri e più inutili» (Serao 1916: 185). A loro suggerisce di lavorare: «non pensate ad altro, non fate altro, lavorate. Dio benedirà l'opera delle vostre mani» (Serao 1916: 187); «fate la calza per i soldati d'Italia» (Serao 1916: 190);

sappiatelo, se non le fate voi, in provincia, le calze di lana per i soldati, care donne, nessuno le farà. Sappiate che le signore e le signorine di città, quelle che lavorano per $\mathrm{i}$ soldati, non fanno calze. Esse fanno altri lavori di lana, con l'uncinetto, coi ferri, ma calze niente. Molte di esse si affannano per trovar modelli di sciarpe, di berretti, di

${ }^{10}$ Durante la prima guerra mondiale e ancor più durante la seconda, donne generalmente altolocate, ma non solo, intrattenevano relazioni epistolari con i soldati impegnati al fronte con lo scopo di far compagnia e alleviare la sofferenza e la solitudine degli uomini. A esse fu dato il nome di madrine dei soldati: «il contatto con la madrina diventa per i soldati un farmaco che lenisce le ferite della guerra. Un rapporto che proprio perché eccede le regole dei rapporti sociali e di genere, può diventare più importante della corrispondenza con la famiglia [...] a lei si può confidare tutto. La paura e l'angoscia» (Molinari 2014: 480). 
puncho, di passamontagna, di guanti e perdono la testa fra i molti, fra i troppi esemplari che sono in circolazione. (Serao 1916: 190-191)

Insomma, per Serao, la cosa giusta che può fare una donna è lavorare a maglia per poter soccorrere i soldati che si trovano al freddo sulle montagne ${ }^{11}$.

Parlare di guerra è l'occasione per la giornalista di offrire al lettore uno spaccato di vita sociale nell'Italia del tempo: ci ricorda che l'analfabetismo era molto diffuso e vi erano «comitati di corrispondenza impiantati nella case signorili» (Serao 1916: 166) dove si recavano «donne mature, perché con tante maestre e tante scuole, diffuse un po' dappertutto, anche nei miseri paeselli, giovani donne analfabete non esistono più» (Serao 1916: 167). Purtroppo è noto che quanto scrive Serao è solo parzialmente vero: nel 1915 l'analfabetismo era ancora molto diffuso. Tullio De Mauro sostiene che «all'inizio del secolo gli analfabeti erano quasi la metà della popolazione e superavano il 69\% nel mezzogiorno» (De Mauro 2001: 91). Secondo un altro studio, la percentuale degli analfabeti nel 1911 (popolazione dai 6 anni in su) era nel Nord del 22.4\%, nel Centro del 41.4 e nel Sud del 58.9, con una percentuale complessiva del 37.9 (Procacci / Farolfi 1984:146).

Con frequenza la giornalista dedica la sua attenzione alle classi meno abbienti: partiti i contadini per il fronte,

le contadine italiane [...] hanno raddoppiato, triplicato il loro lavoro quotidiano: le più pesanti, le più dure, le più estenuanti fatiche degli uomini, esse le hanno assunte [...] la contadina italiana lavorava la terra come un uomo. (Serao 1916: 115-116)

La «vigorosa lavoratrice dei campi» (Serao 1916: 144) dunque lavora ma anche le «piccole borghesi impiegate sono tornate all'antico impiego» (Serao 1916: 145). Benché concluda l'articolo scrivendo «il mondo non se ne avvede, il mondo non sa il cuore che esse hanno, nel loro tacito sacrificio» (Serao 1916: 145), infastidisce il suo aristocratico distacco nei confronti di chi è meno privilegiata di lei, così come infastidisce il tono paternalistico adottato.

Nei suoi articoli si occupa anche di argomenti meno impegnati e decisamente frivoli, come la moda. Ma proprio «attraverso le peggiori banalità, rivive

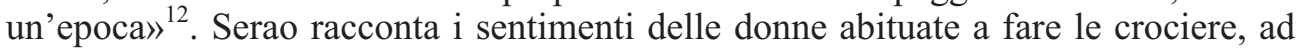
andare in Engadina a sciare, ad andare a Parigi in primavera: «è infelicissima in questo momento perché non può più solcare $\mathrm{i}$ mari, diventati troppo pericolosi. La guerra cambia i destini di migliaia di persone»(Serao 1916: 155). Di fronte alla

${ }^{11}$ Fussell cita un saggio intitolato What to send 'Your Soldiers' in cui si consiglia di inviare agli uomini al fronte caramelle dette bulls' eyes: «I Bulls' eyes devono essere pieni di menta, perché è la menta che nelle notti fredde tiene caldi quelli che la succhiano. Essa ha inoltre un effetto digestivo, anche se ciò ha poca importanza al fronte, dove la salute è così buona e ben difficilmente si sente parlare di indigestione» (Fussell 2000: 112).

${ }^{12}$ Lo pensa Marie-Gracieuse Martin-Gistucci nella prefazione all'edizione critica da lei curata del romanzo incompiuto di Matilde Serao L'ebbrezza, il servaggio e la morte. 
riapertura dei teatri a Parigi, dove tra l'altro "sono scomparsi tutti i drammi, tutte le commedie, tutte le riviste a base patriottica», Serao si domanda se sia giusto o no allontanare dalle menti il conflitto che infuria; sebbene non sia esplicita la risposta, tra le righe ci dice che, nonostante gli spettacoli teatrali tentino di ridare spensieratezza, i fatti bellici (a p. 304 allude alla battaglia di Verdun) riportano alla dura realtà. A proposito di moda, biasima l'abbigliamento di alcune donne, soprattutto di alcune vedove (che definisce «merry widows»): «molte vedove di ufficiali e di soldati morti in guerra indossano vesti vistose ed hanno un contegno che stupisce e indigna» (Serao 1916: 204-205). Il loro «lutto provocante» si esplicita in

un vestito il cui corsage mostra il collo nudo sino quasi al cavo dello stomaco, sotto un largo colletto bianco arrovesciato, mentre la gonna, rotonda e corta, mostra gli stivalini allacciati sino a metà gamba, mentre un cappellino civettuolo oltre ogni dire, col grande velo nero pendente dietro, posa sovra i capelli biondi ossigenati. (Serao 1916: 205)

In un articolo scritto posteriormente e datato Gennaio 1916, dopo aver definito «grottesca mascheratura a cui i grandi sarti francesi hanno invitato le donne, e le donne subito hanno accettato il perfido invito» (Serao 1916: 300), si domanda:

non si poteva creare una moda semplice, sobria, modesta, fatta, diciamo così, per le donne dei paesi belligeranti, una moda che non fosse in uno stridente disaccordo con le pene segrete di cui dev'essere tormentato ogni cuore femminile, nei paesi in guerra? (Serao 1916: 301)

\section{CONCLUSIONI}

Come si è visto, l'intento di Matilde Serao in Parla una donna non è tanto quello di occuparsi degli aspetti politico-militari legati al conflitto quanto quello di raccogliere testimonianze: leggere le pagine di Matilde Serao sulla guerra rappresenta un modo singolare per ricostruire lo spirito del tempo in cui tali esperienze ebbero corso. La modalità con la quale si svolse questa guerra portò cambiamenti epocali, che Fulvio Senardi sintetizza così:

tutti gli studiosi concordano sull'inaudita novità di un conflitto che, in modo tanto drastico quanto emblematico, pare concludere il XIX secolo, trascinando con sé nell'abisso l'ottimismo della Belle Epoque. (Senardi 2008: 7)

Sappiamo che Serao era contraria alla guerra, lo ribadisce nel 1926 quando pubblica Mors tua dove polemizza contro la guerra del '15-'18 e contro ogni guerra. È stato detto che il suo era un pacifismo di tipo umanitario, non politico e, se accetta la guerra, lo fa solo per dovere patriottico. Secondo Buzzi, «Serao, che non è mai stata bellicista, paga l'indispensabile tributo alla retorica patriottarda e alle menzogne ufficiali» (Buzzi 1981: 125). Non essendo testimone oculare, non 
può garantire la presa diretta sul reale: il risultato è una visione distorta, eroicizzante e retorica del conflitto. Sono proprio la retorica e il sentimentalismo a guastare pagine interessanti (Rasy (1994: 244) parla di «irritante leggerezza patriottica»); si veda ad esempio questa affermazione dove, parlando delle donne, si spinge a fare un'affermazione poco credibile: «per loro era cosa naturalissima che $\mathrm{i}$ figli, unico sostegno di quelle grame esistenze, servissero la patria» (Serao 1916: 95).

Serao scrive per un pubblico particolare: quello femminile col quale è in sintonia. Benedetto Croce la considerava scrittrice della piccola borghesia e della plebe. In effetti, benché presenti una vasta tipologia femminile - dalla popolana, alla borghese, all'aristocratica, alla ragazza da marito, alla madre angosciata -, Serao si rivolge a un pubblico femminile di classe media. In Parla una donna vi è sempre il coinvolgimento personale: i suoi occhi di «lavoratrice della penna» vanno oltre il dato oggettivo; nei suoi scritti è indubbia la grande capacità di osservare e interpretare il reale, di partecipare alle miserie e ai dolori delle donne e, nel difendere la donna lavoratrice, testimonia la sua condizione. Come ha scritto Mariarosaria Olivieri:

pur prendendo in esame la tragica condizione delle lavoratrici donne, invia in realtà il suo messaggio ad un più vasto pubblico, prevalentemente maschile, formato dai lettori del quotidiano. Cosicché, tanto gli scritti giornalistici quanto le opere narrative della Serao, escono fuori dall'ambito di una letteratura femminile per entrare in quello più vasto di una letteratura opera di donne. (Olivieri 1990: 14)

In questa analisi è stata però messa in luce anche la posizione conservatrice del ruolo della donna vista esclusivamente come madre e moglie, in posizione ancillare rispetto all'uomo. Risulta però difficilmente accettabile il bipolarismo tra la negazione dell'emancipazione della donna e l'esaltazione della libertà e dell'autonomia della donna.

Scrive Anna Banti, «osservare, accumulare figure, ambienti, fatti, gesti, parole [...] rimane il suo vero scopo» (Banti 1965: 31). La sua lingua immediata, mimetica, schietta, antiletteraria, la sua espressività libera da assilli stilistici assicura una prontezza comunicativa non comune; del resto è la stessa Serao a sostenere: «io credo con la vivacità di quel linguaggio incerto e di quello stile rotto di infondere nelle opere mie il calore» (Ojetti 1946: 276). Certo, come detto in precedenza, certe impennate retoriche, certe melensaggini, certi eccessi verbali, la mancanza di rigore metodologico guastano molte pagine; in ogni modo Parla una donna, pur nei suoi limiti, è un testo che va annoverato tra gli scritti che documentano da una particolare angolatura il contesto attorno al quale si svolse la Grande guerra.

\section{RIFERIMENTI BIBLIOGRAFICI}

BANTI, Anna (1965): Matilde Serao, Torino, UTET. 
BERTACCHINI, Renato (1966): «Guerra e letteratura. Rassegna di antologie e ristampe relative alla prima guerra mondiale», Studium 62 (luglio), pp. 472-482.

BUZZI, Giancarlo (1981): Invito alla lettura di Matilde Serao, Milano, Mursia.

DE MAURO, Tullio (2001): Storia linguistica dell'Italia unita, Bari-Roma, Laterza.

DE NUNZIO SCHILARDI, Wanda (1986): Matilde Serao giornalista, Lecce, Milella.

De NunZIO Schilardi, Wanda (2004): L'invenzione sul reale. Studi su Matilde Serao, Bari, Palomar.

De NunZIo SchILARDI, Wanda (2005): «La settimana di Matilde Serao», Rivista di letteratura italiana, XXIII/1-2, pp. 115-124.

DonNa PaOla [Paola Baronchelli Grosson] (1915): La funzione della donna in tempo di guerra, Firenze, Bemporad \& Figlio Editori.

FRESCHI, Ombretta (2013): «Donna in trincea. Flavia Steno al fronte (1915-1918)», Nuova corrente, 151/LX, pp. 59-72.

Fussell, Paul $\left(2000^{2}\right)$ : La Grande guerra e la memoria moderna, Bologna, Il Mulino. [Traduzione italiana di Giuseppe Panzieri di The Great War and Modern Memory, Oxford, Oxford University Press, 1975]

GADDA, Carlo Emilio (1965): Giornale di guerra e prigionia, Torino, Einaudi.

GIBELLI, Antonio (2002): «Nefaste meraviglie. Grande guerra e apoteosi della modernità», in: Walter Barberis (a cura di), Storia d'Italia, Annali 18, Guerra e pace (ottava edizione), Torino, Einaudi, pp. 569-571.

GIBELLI, Antonio (2003): L'officina della Grande Guerra, Torino, Bollati Boringhieri.

MASINI, Piercarlo (1965): «L'emancipazione della donna in una polemica tra Anna Maria Mozzoni e Matilde Serao», Critica sociale, 20 maggio, a. 57/10, pp. 251253.

MOLINARI, Augusta (2014): «Scritture femminili della Grande Guerra: il caso italiano», in Antonio Castillo Gómez, Verónica Sierra Blas (eds), CartasLettres-Lettere. Discursos, prácticas y representaciones epistolares (siglos XIVXX), Alcalá, Universidad de Alcalá Servicio de Publicaciones, pp. 465-481.

OJETTI, Ugo (1946): Alla scoperta dei letterati, Firenze, Le Monnier.

OlivierI, Mariarosaria (1990): Tra libertà e solitudine. Saggi su letteratura e giornalismo femminile: Matilde Serao, Sibilla Aleramo, Clotilde Marghini, Pisa, Edizione dell'Ateneo.

PANNUNZIO, Giorgio (2000): «Sulle novelle belliche di Federico De Roberto», Studi Medioevali e Moderni 2, pp. 119-150.

PrEZZOLINI, Giuseppe (1919): Dopo Caporetto, Roma, La Voce.

PROCACCI, Giuliano / FAROLFI, Bernardino (1984): 50 generazioni: corso di storia, volume III, Firenze, La Nuova Italia.

RASY, Elisabetta (1994): «Parla una donna. Il diario di guerra di Matilde Serao», Chroniques italiennes 39-40, pp. 243-254.

Salsa, Carlo (1982): Trincee. Confidenze di un fante, Milano, Mursia. [Originariamente: Milano, Sonzogno, 1927].

SCAPPATICCI, Tommaso (1995): «Il pubblico della Serao», Problemi 103, settembre-dicembre, pp. 268-297 
SENARDI, Fulvio (a cura di) (2008): Scrittori in trincea. La letteratura e la grande guerra, Roma, Carocci.

SERAO, Matilde (1916): Parla una donna. Diario femminile di guerra. Maggio 1915-Marzo 1916, Milano, Treves.

SERAO, Matilde (1938): «A furia di urti e gomitate. Lettera a Gaetano Bonavenia», Nuova Antologia, 16 agosto 1938, pp. 402-412.

SERAO, Matilde (1977): L'ebbrezza, il servaggio e la morte, prefazione a cura di Marie-Gracieuse Martin-Gistucci, Napoli, Guida editore.

SERAO, Matilde (2014): Parla una donna, introduzione di Nunzia Soglia, Salerno, Il Grappolo.

SERRA, Renato (1974): Scritti letterari, morali e politici: saggi e articoli del 1900 al 1915, a cura di Mario Isnenghi, Torino, Einaudi.

TROTTA, Donatella (2008): La via della penna e dell'ago. Matilde Serao tra giornalismo e letteratura con antologia di scritti rari e immagini, Napoli, Liguori editore. 